\title{
Huge Hepatic Angiomyolipoma Mimicking Low Grade Hepatocellular Carcinoma
}

\author{
Hyeo Seong Hwang, Dae Hoon Han \\ Department of Surgery, Yonsei University College of Medicine, Seoul, Korea
}

Received Dec. 31, 2020

Revised Jan. 29, 2021

Accepted Feb. 1, 2021
A 41-year-old man was diagnosed with a huge symptomatic liver mass and was referred to our hospital for liver biopsy and further evaluation. He presented with right upper quadrant tenderness. Enhanced abdominal computed tomography and magnetic resonance imaging revealed a $12.5-\mathrm{cm}$ relatively well-defined heterogeneous enhancing mass in the right inferior liver with a large exophytic component containing a fat component and progressive delayed enhancement. The patient underwent right inferior sectionectomy. The pathological diagnosis was confirmed as angiomyolipoma, $12.3 \times 9.2 \times 5.0 \mathrm{~cm}$ in size, with tumor necrosis in $20 \%$ of the tissue. Hepatic angiomyolipoma is known as a benign tumor, but in our case, because of the large tumor size and coagulative necrosis, this tumor had malignant potential; surgical resection was deemed to be appropriate, and close follow-up monitoring was essential postoperatively. (J Liver Cancer 2021;21:76-80)

Keywords: Hepatic angiomyolipoma; Hepatocellular carcinoma

\section{INTRODUCTION}

Hepatic angiomyolipoma (AML) is the second most common type of AML after renal AML. AML is a rare mesenchymal liver tumor composed of mature adipocytes, blood vessels, and spindle-epithelioid cells. Therefore, hepatic AML is classified into four subtypes according to the differentiation line and components: mixed, lipomatous, myomatous, and angiomatous. ${ }^{1}$ The various and dynamic component patterns of AML on computed tomography (CT) or magnetic resonance imaging (MRI) result in an inconclusive diagnosis or misdiagnosis of the tumor as a hepatocellular carcinoma. In

\footnotetext{
Corresponding author: Dae Hoon Han

Department of Surgery, Yonsei University College of Medicine, 50-1 Yonsei-ro, Seodaemun-gu, Seoul 03722, Korea

Tel. +82-2-2228-2100, Fax. +82-2-313-8289

E-mail; DHHAN@yuhs.ac

https://orcid.org/0000-0003-2787-7876
}

2017, Klompenhouwer reviewed 18 studies reporting on 292 patients and suggested liver biopsy to diagnose hepatic AML in case of inconclusive imaging findings. Annual imaging follow-up is acceptable in cases of clear hepatic AML on imaging. However, when the patient is symptomatic, biopsy results are uncertain, or if the tumor size is large, resection should be considered. ${ }^{2}$ AML $>10 \mathrm{~cm}$ in size with cytologic atypia or coagulative necrosis may have malignant potential. ${ }^{3}$ Coagulative necrosis is characterized by a cluster of pleomorphic cells in which eosinophilic granular cytoplasm and vesicular nuclei are found. ${ }^{4}$ Herein, we present the case of a patient with AML $(>12 \mathrm{~cm})$ with malignant potential who underwent surgical resection rather than a liver biopsy considering patient safety. 


\section{CASE REPORT}

\section{Clinical findings}

A 41-year-old man visited a local clinic for abdominal dis- comfort and pain. He was diagnosed with a huge symptomatic liver mass and was referred to our hospital for liver biopsy and further evaluation. He presented with right upper quadrant tenderness, and on physical examination, the abdominal mass was palpated. He had been healthy without


Figure 1. Abdominal computed tomography revealed a $12.5-\mathrm{cm}$ sized relatively well-defined heterogeneous enhancing mass in the right inferior liver with a large exophytic component containing a fat component $(A, B)$.
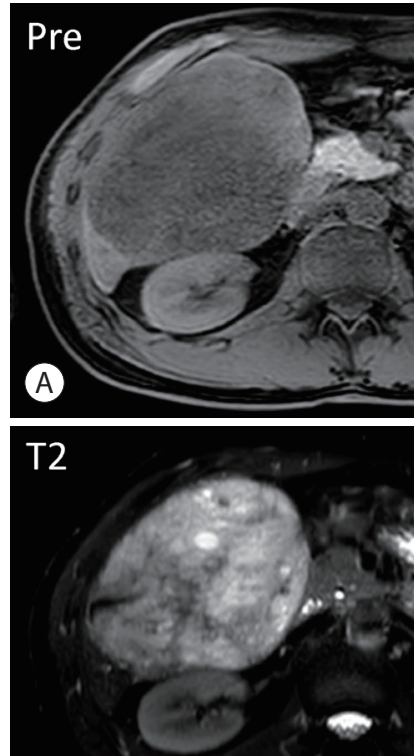

(E)

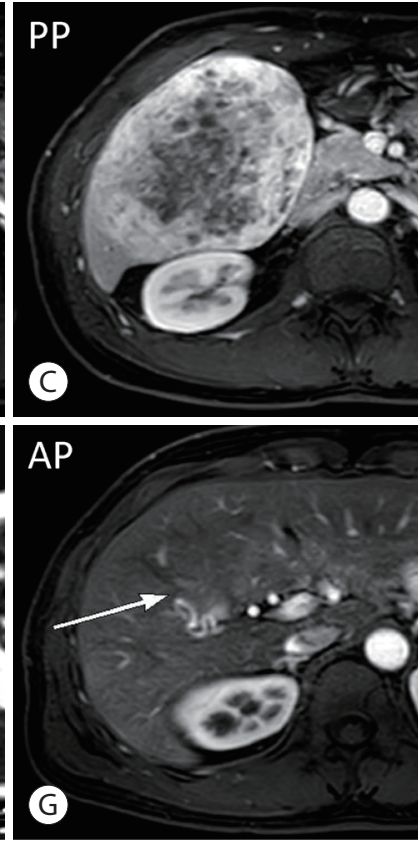
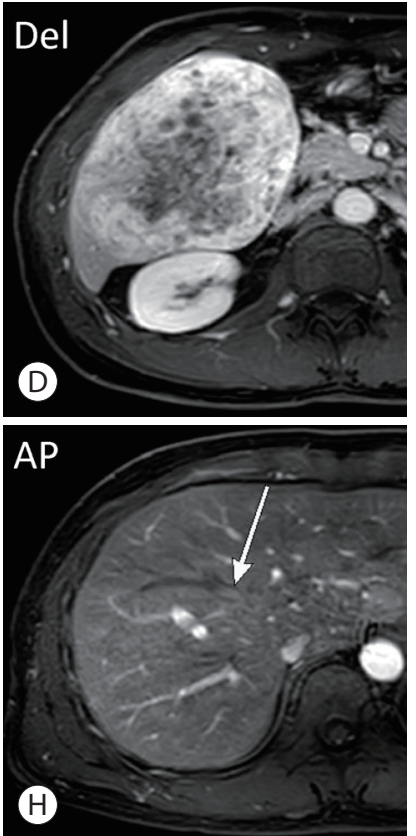

Figure 2. On magnetic resonance imaging, the hepatic mass showed progressive delayed enhancement; the last two arterial phase images revealed the presence of early draining veins around the tumor (arrows), which is a typical imaging finding of angiomyolipoma (A-H). AP, arterial phase; PP, portal phase; PDFF, proton density fat fraction. 
any medical or surgical history. Initial laboratory findings on admission were all confirmed to be within normal limits, including tumor markers (alpha-fetoprotein, $4.36 \mathrm{ng} / \mathrm{mL}$; pro- tein induced by vitamin $\mathrm{K}$ absence II, $31 \mathrm{mAU} / \mathrm{mL}$ ). Hepatitis $B$ surface antigen and hepatitis $C$ virus antibody tests were negative, and he was neither a smoker nor an alcoholic.
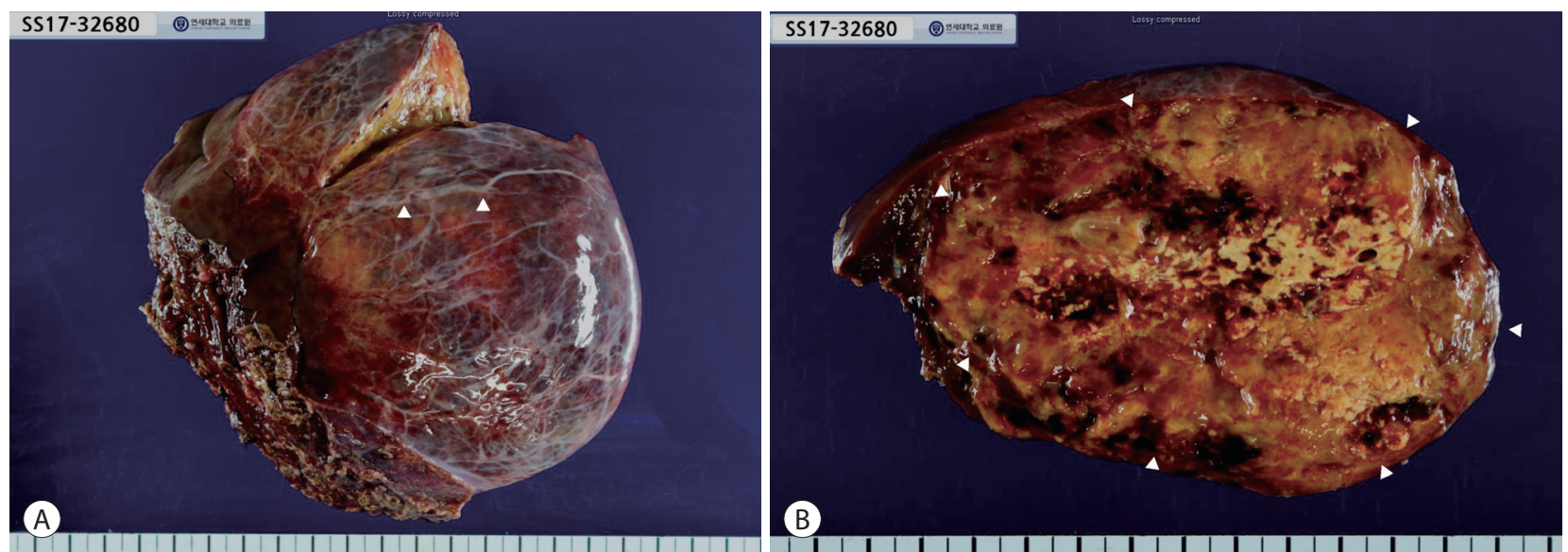

Figure 3. (A) Gross image of specimen with dilated veins (arrowheads) on liver surface. (B) Resected specimen with heterogeneous fatty and hemorrhagic components (arrowheads).


Figure 4. Histopathological findings of angiomyolipoma; (A) thick-walled vessels (white arrow, portal vein) and thin-walled vessels (black arrow) are fused at the boundary between normal liver tissue $(N)$ and the tumor $(T)(\times 40$, hematoxylin and eosin [H\&E] stain). (B-D) The pleomorphism of histological features of hepatic angiomyolipoma is showed ( $\times 100, \mathrm{H} \& \mathrm{E}$ stain) ( $B$, adipose tissues; $C$, foam cells; and D, smooth muscle cells). 


\section{Imaging findings}

An initial enhanced abdominal CT scan revealed a 12.5$\mathrm{cm}$ relatively well-defined heterogeneous enhancing mass in the right inferior liver with a large exophytic component containing a fat component and showing progressive delayed enhancement. MRI was performed, and Meglumine gadoterate was injected intravenously as a bolus (Fig. 1,2). Positron emission tomography-computed tomography was also performed; however, the preoperative imaging findings could not clearly rule out a malignant tumor.

\section{Diagnosis and treatment}

The patient underwent right inferior sectionectomy with cholecystectomy on June 5th, 2017. The operation lasted 240 minutes, with an estimated gross blood loss of approximately $200 \mathrm{~mL}$; no specific features of cirrhotic liver were found. There was no report of postoperative complications such as hepatic insufficiency, bleeding, bile leakage, or infection. The liver function recovered to within normal limits, and the postoperative CT scan revealed no remarkable complications on postoperative day 5 . The patient was discharged on the 8th postoperative day. During 34 months of follow-up, there was no evidence of tumor recurrence or distant metastasis. In the immunohistochemical analysis, the tumor cells showed positive immunostaining for human melanoma black (HMB)-45. On pathological examination, the tumor was diagnosed as AML, $12.3 \times 9.2 \times 5.0 \mathrm{~cm}$ in size, with tumor necrosis in $20 \%$ of the tissue (Fig. 3). In the arterial phase of MRI, the contrast agent was found to be rapidly exiting the veins around the tumor. Due to the pleomorphism of histological features of hepatic AML, it would have been inappropriate to diagnose AML using needle biopsy (Fig. 4).

\section{DISCUSSION}

AML is a tumor most commonly found in the kidney with the liver being the second-most commonly affected organ. Since its first report by Ishak, ${ }^{5}$ hepatic AML has been shown to have various and dynamic component patterns according to the differentiation line and components and characteristic imaging findings on CT, MRI, and angiography. ${ }^{6,7}$ These patterns of AML on CT and MRI result in the tumor being misdiagnosed as hepatocellular carcinoma. Zhong and $\mathrm{Ji}^{8}$ reported that among 14 cases of hepatic AML, all cases were misdiagnosed preoperatively as sarcoma, hepatocellular carcinoma, or other benign tumors. In addition, because of the pleomorphism of the histological features, diagnosis based on needle biopsy findings may be inappropriate. In our case, the patient was recommended for needle biopsy at the first hospital he visited even though his tumor was larger than 12 $\mathrm{cm}$ in size. However, it would have been difficult to obtain sufficient components to confirm AML using fine needle biopsy because $20 \%$ of the tumor was necrotic and the four components were not evenly distributed. In preoperative MRI, early venous return in the tumor was found, which is a typical characteristic of hepatic AML. However, if the tumor is not sufficiently large, this phenomenon is difficult to identify in actual clinical practice. ${ }^{9,10}$ Regarding pathological findings, the reactivity of tumor cells with the melanoma-specific antibody, HMB-45, can accurately diagnose AML. ${ }^{11}$ Moreover, there are few reports of malignant hepatic AML with metastasis and these malignant hepatic AMLs possess the following characteristics: tumor size $>10 \mathrm{~cm}$, cytologic atypia, and coagulative necrosis. ${ }^{3,12,13}$ Our patient was not diagnosed with hepatocellular carcinoma; however, because of the large tumor size and coagulative necrosis at the center of the mass, the tumor had malignant potential. Therefore, surgical resection was deemed appropriate, and close postoperative follow-up monitoring was essential.

\section{Conflicts of Interest}

The authors have no financial conflicts of interest.

\section{REFERENCES}

1. Wang CP, Li HY, Wang H, Guo XD, Liu CC, Liu SH, et al. Hepatic angiomyolipoma mimicking hepatocellular carcinoma: magnetic resonance imaging and clinical pathological characteristics in 9 cases. Medicine (Baltimore) 2014;93:e194.

2. Klompenhouwer AJ, Verver D, Janki S, Bramer WM, Doukas M, Dwarkasing RS, et al. Management of hepatic angiomyolipoma: a 
systematic review. Liver Int 2017;37:1272-1280.

3. Nguyen TT, Gorman B, Shields D, Goodman Z. Malignant hepatic angiomyolipoma: report of a case and review of literature. Am J Surg Pathol 2008:32:793-798.

4. Sato K, Ueda Y, Tachibana H, Miyazawa K, Chikazawa I, Kaji S, et al. Malignant epithelioid angiomyolipoma of the kidney in a patient with tuberous sclerosis: an autopsy case report with p53 gene mutation analysis. Pathol Res Pract 2008;204:771-777.

5. Ishak KG. Mesenchymal tumor of the liver. In. Okuda K, Peters RL, eds. Hepatocellular carcinoma. New York; Wiley Medical, 1976:247-304.

6. Chang JC, Lee YW, Kim HJ. Preoperative diagnosis of angiomyolipoma of the liver. Abdom Imaging 1994;19:546-548.

7. Blumgart R, Payne M, Rhodes A. Angiomyolipoma of the liver. Clin Radiol 1987;38:329-330.

8. Zhong DR, Ji XL. Hepatic angiomyolipoma-misdiagnosis as hepa- tocellular carcinoma: a report of 14 cases. World J Gastroenterol 2000;6:608-612.

9. Kiuchi R, Sakaguchi T, Kitajima R, Furuhashi S, Takeda M, Hiraide $T$, et al. Hepatic angiomyolipoma with early drainage veins into the hepatic and portal vein. Clin J Gastroenterol 2019;12:361-366.

10. Saito $Y$, Shimada M, Utsunomiya $T$, Morine $Y$, Imura $S$, Ikemoto $\mathrm{T}$, et al. Hepatic epithelioid angiomyolipoma with arterioportal venous shunting mimicking hepatocellular carcinoma: report of a case. J Med Invest 2013;60:262-266.

11. Nonomura A, Mizukami Y, Kadoya M, Matsui O, Shimizu K, Izumi R. Angiomyolipoma of the liver: its clinical and pathological diversity. J Hepatobiliary Pancreat Sur 1996;3:122-132.

12. Nonomura A, Mizukami Y, Kadoya M. Angiomyolipoma of the liver: a collective review. J Gastroenterol 1994;29:95-105.

13. Yang $C Y$, Ho MC, Jeng YM, Hu RH, Wu YM, Lee PH. Management of hepatic angiomyolipoma. J Gastrointest Surg 2007;11:452-457. 